\author{
OAK RIDGE \\ NATIONAL LABORATORY
}

MANAGED BY UT-BATTELLE

FOR THE DEPARTMENT OF ENERGY
ORNL/TM-2008/132

\section{Data Compilation for AGR-2 Baseline Coated Particle Batch G73J-14-93071A}

John D. Hunn

October 2008

This report was prepared as an account of work sponsored by an agency of the United States Government. Neither the United States Government nor any agency thereof, or any of their employees, makes any warranty, expressed or implied, or assumes any legal liability or responsibility for any third party's use, or the results of such use, of any information, apparatus, product or process disclosed in this report, or represents that its use by such third party would not infringe privately owned rights. 


\section{DOCUMENT AVAILABILITY}

Reports produced after January 1, 1996, are generally available free via the U.S. Department of Energy (DOE) Information Bridge.

Web site http://www.osti.gov/bridge

Reports produced before January 1, 1996, may be purchased by members of the public from the following source.

National Technical Information Service

5285 Port Royal Road

Springfield, VA 22161

Telephone 703-605-6000 (1-800-553-6847)

TDD 703-487-4639

Fax 703-605-6900

E-mail info@ntis.fedworld.gov

Web site http://www.ntis.gov/support/ordernowabout.htm

Reports are available to DOE employees, DOE contractors, Energy Technology Data Exchange (ETDE) representatives, and International Nuclear Information System (INIS)

representatives from the following source.

Office of Scientific and Technical Information

P.O. Box 62

Oak Ridge, TN 37831

Telephone 865-576-8401

Fax 865-576-5728

E-mail reports@adonis.osti.gov

Web site http://www.osti.gov/contact.html

This report was prepared as an account of work sponsored by an agency of the United States Government. Neither the United States Government nor any agency thereof, nor any of their employees, makes any warranty, express or implied, or assumes any legal liability or responsibility for the accuracy, completeness, or usefulness of any information, apparatus, product, or process disclosed, or represents that its use would not infringe privately owned rights. Reference herein to any specific commercial product, process, or service by trade name, trademark, manufacturer, or otherwise, does not necessarily constitute or imply its endorsement, recommendation, or favoring by the United States Government or any agency thereof. The views and opinions of authors expressed herein do not necessarily state or reflect those of the United States Government or any agency thereof. 


\title{
Data Compilation for AGR-2 Baseline Coated Particle Batch G73J-14-93071A
}

\author{
John D. Hunn \\ Oak Ridge National Laboratory
}

Coated particle fuel batch G73J-14-93071A was produced by the Babcock and Wilcox Company $(\mathrm{B} \& \mathrm{~W})$ for possible selection as UCO baseline fuel for the Advanced Gas Reactor Fuel Development and Qualification Program's AGR-2 irradiation test. Batch G73J-14-93071A is a single batch of TRISO-coated $425 \mu \mathrm{m}$ nominal diameter $14 \%$ low enrichment uranium oxide/uranium carbide kernels (LEUCO). The TRISO-coatings consist of a 50\% dense carbon buffer layer (100 $\mu \mathrm{m}$ nominal thickness) followed by a dense inner pyrocarbon layer (40 $\mu \mathrm{m}$ nominal thickness) followed by a SiC layer (35 $\mu$ m nominal thickness) followed by another dense outer pyrocarbon layer (40 $\mu \mathrm{m}$ nominal thickness).

The AGR-2 Fuel Specification (INL SPC-923) provides the requirements necessary for acceptance of the fuel manufactured for the AGR-2 irradiation test. The bulk of the kernels and coated particle acceptance testing was performed at B\&W and is not contained in this report. Sample NP-B7927 was sent to ORNL for supplemental characterization. The procedures for the limited characterization and qualification of the particles performed at ORNL are outlined in ORNL product inspection plan AGR-CHAR-PIP-09. The BAFo equivalent optical anisotropies of the inner and outer pyrocarbon layers are reported on Inspection Report Form IRF-09, with a determination as to whether the particle batch satisfied the specified parameters for this property. The batch was found to satisfy the AGR-2 Fuel Specification SPC-923, Rev. 1 for IPyC and OPyC anisotropy.

Also provided in this data package are data on the true BAFo, average particle weight, OPyC open porosity, and $\mathrm{SiC}$ soot inclusion defect fraction. True BAFo is calculated as $(1+\mathrm{N}) /(1-\mathrm{N})$, where $\mathrm{N}$ is the diattenuation. This differs from equivalent $\mathrm{BAFO}=1+3 \mathrm{~N}$, which is the calculation used by the fuel specification to allow comparison to historical measurements. Average OPyC open porosity was determined using a single sample to be $0.65 \mathrm{ml} / \mathrm{m}^{2}$. An unusually high $\mathrm{SiC}$ soot defect fraction was observed in this sample, apparently due to a fabrication anomaly which resulted in one of the hot sampling cups disturbing the bed fluidization during $\mathrm{SiC}$ deposition. This is discussed further in the pages following Data Report Form DRF-32. 


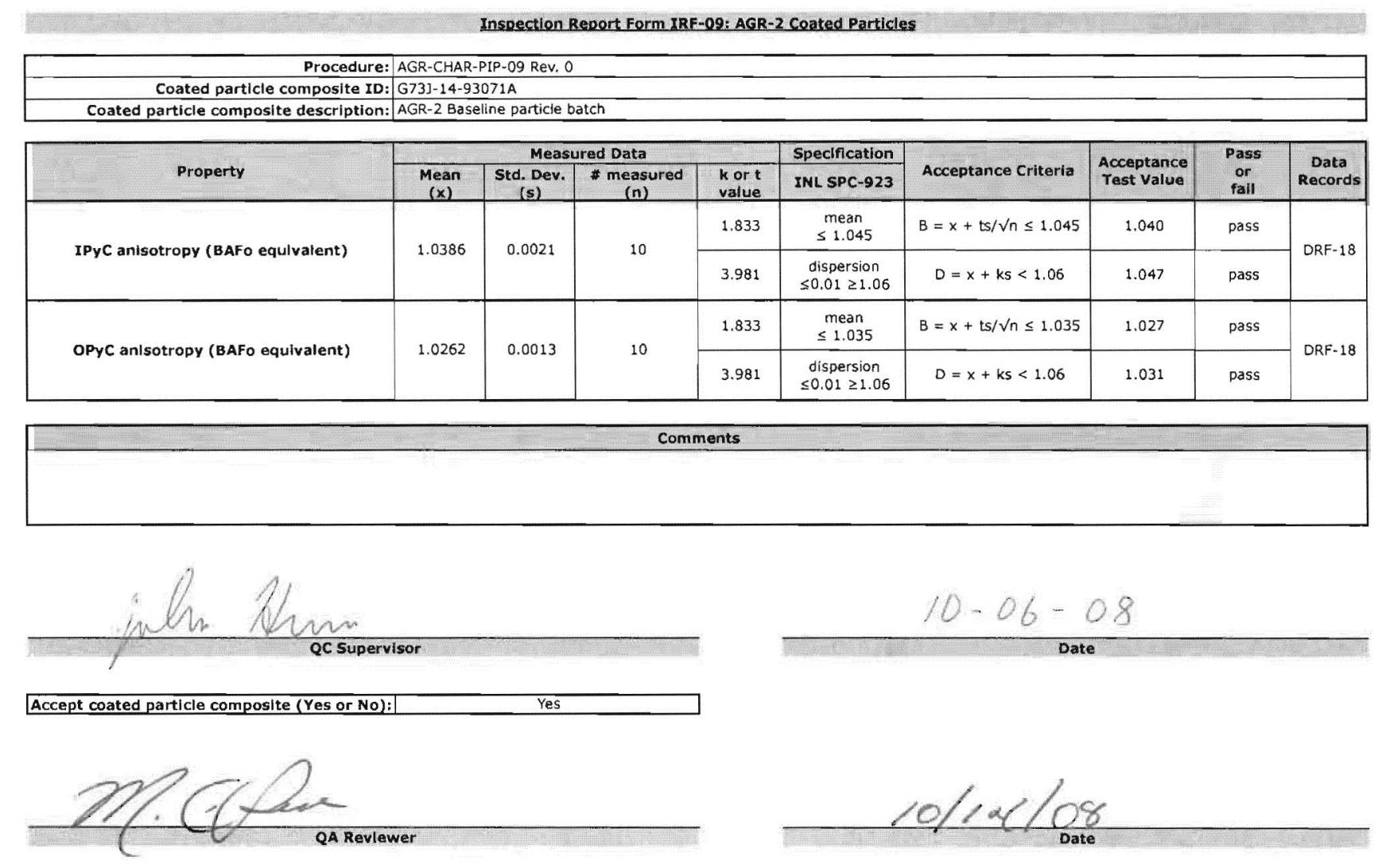


Data Report Form DRF-18A: Measurement of Pyrocarbon Anisotropy using the 2-MGEM - IPyC

\begin{tabular}{|c|c|}
\hline Procedure: & AGR-CHAR-DAM-18 Rev, 1 \\
\hline Operator: & G. E. Jellison \\
\hline Mount ID: & M08082501 \\
\hline Sample ID: & NP-B7927-B01 \\
\hline Sample Description: & From G73J-14-93071A AGR-2 Baseline batch \\
\hline Folder containing data: & 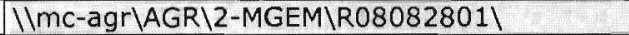 \\
\hline
\end{tabular}

\begin{tabular}{|c|c|c|c|c|c|c|c|}
\hline \multirow{2}{*}{ Particle \# } & \multirow{2}{*}{$\begin{array}{c}\text { Grid } \\
\text { Position }\end{array}$} & \multicolumn{3}{|c|}{ Diattenuation } & \multicolumn{3}{|c|}{ Equivalent $\mathrm{BAFO}=1+3 \mathrm{~N}$} \\
\hline & & Average & St. Dev. & Ave. Error & Average & St. Dev. & Ave. Error \\
\hline 1 & 4,4 & 0.0127 & 0.0023 & 0.0004 & 1.0381 & 0.0069 & 0.0012 \\
\hline 2 & 4,5 & 0.0116 & 0.0022 & 0.0004 & 1.0348 & 0.0066 & 0.0012 \\
\hline 3 & 4,6 & 0.0130 & 0.0021 & 0.0004 & 1.0390 & 0.0063 & 0.0012 \\
\hline 4 & 5,3 & 0.0141 & 0.0022 & 0.0004 & 1.0423 & 0.0066 & 0.0012 \\
\hline 5 & 5,5 & 0.0133 & 0.0025 & 0.0005 & 1.0399 & 0.0075 & 0.0015 \\
\hline 6 & 5,6 & 0.0123 & 0.0024 & 0.0005 & 1.0369 & 0.0072 & 0.0015 \\
\hline 7 & 6,4 & 0.0131 & 0.0024 & 0.0005 & 1.0393 & 0.0072 & 0.0015 \\
\hline 8 & 6,5 & 0.0131 & 0.0023 & 0.0005 & 1.0393 & 0.0069 & 0.0015 \\
\hline 9 & 6,6 & 0.0132 & 0.0025 & 0.0004 & 1.0396 & 0.0075 & 0.0012 \\
\hline 10 & 5,7 & 0.0121 & 0.0024 & 0.0005 & 1.0363 & 0.0072 & 0.0015 \\
\hline \multicolumn{2}{|c|}{ Average } & 0.0129 & 0.0023 & 0.0005 & 1.0386 & 0.0070 & 0.0014 \\
\hline
\end{tabular}

Mean of average BAFo per particle: 1.0386 Standard deviation of average BAFo per particle: 0.0021

\section{Comments}

Run stopped after particle 4. Particles 5-10 use file labels 01a-06a
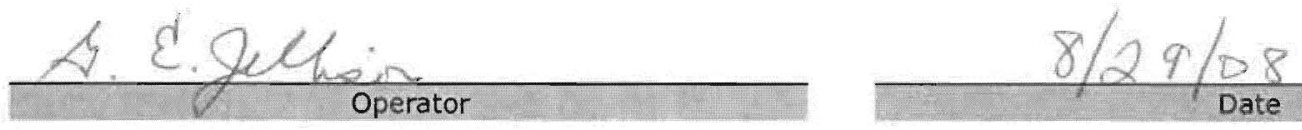
Data Report Form DRF-18B: Measurement of Pyrocarbon Anisotropy using the 2-MGEM - OPYC

\begin{tabular}{|r|l|}
\hline Procedure: & AGR-CHAR-DAM-18 Rev. 1 \\
\hline Operator: & G. E. Jellison \\
\hline Mount ID: & M08082501 \\
\hline Sample ID: & NP-B7927-B01 \\
\hline Sample Description: & From G73J-14-93071A AGR-2 Baseline batch \\
\hline Folder containing data: & IImc-agr\AGR\2-MGEM\R08082801\} $\\
{\hline}$
\end{tabular}

\begin{tabular}{|c|c|c|c|c|c|c|c|}
\hline \multirow{2}{*}{ Particle \# } & \multirow{2}{*}{$\begin{array}{c}\text { Grid } \\
\text { Position }\end{array}$} & \multicolumn{3}{|c|}{ Diattenuation } & \multicolumn{3}{|c|}{ Equivalent BAFo $=1+3 \mathrm{~N}$} \\
\hline & & Average & St. Dev. & Ave. Error & Average & St. Dev. & Ave. Error \\
\hline 1 & 4,4 & 0.0089 & 0.0021 & 0.0004 & 1.0267 & 0.0063 & 0.0012 \\
\hline 2 & 4,5 & 0.0088 & 0.0020 & 0.0004 & 1.0264 & 0.0060 & 0.0012 \\
\hline 3 & 4,6 & 0.0079 & 0.0020 & 0.0004 & 1.0237 & 0.0060 & 0.0012 \\
\hline 4 & 5,3 & 0.0088 & 0.0020 & 0.0004 & 1.0264 & 0.0060 & 0.0012 \\
\hline 5 & 5,5 & 0.0086 & 0.0023 & 0.0005 & 1.0258 & 0.0069 & 0.0015 \\
\hline 6 & 5,6 & 0.0087 & 0.0022 & 0.0005 & 1.0261 & 0.0066 & 0.0015 \\
\hline 7 & 6,4 & 0.0087 & 0.0027 & 0.0005 & 1.0261 & 0.0081 & 0.0015 \\
\hline 8 & 6,5 & 0.0097 & 0.0021 & 0.0004 & 1.0291 & 0.0063 & 0.0012 \\
\hline 9 & 6,6 & 0.0086 & 0.0021 & 0.0005 & 1.0258 & 0.0063 & 0.0015 \\
\hline 10 & 5,7 & 0.0085 & 0.0020 & 0.0005 & 1.0255 & 0.0060 & 0.0015 \\
\hline \multicolumn{2}{|c|}{ Average } & 0.0087 & 0.0022 & 0.0005 & 1.0262 & 0.0065 & 0.0014 \\
\hline
\end{tabular}

Mean of average BAFo per particle: 1.0262 Standard deviation of average BAFo per particle: 0.0013

\section{Comments}
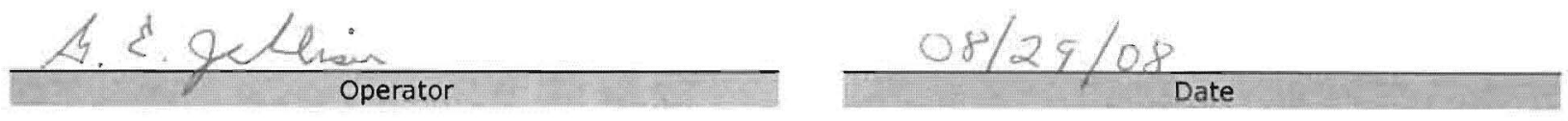
Data Report Form DRF-18A: Measurement of Pyrocarbon Anisotropy using the 2-MGEM - IPYC

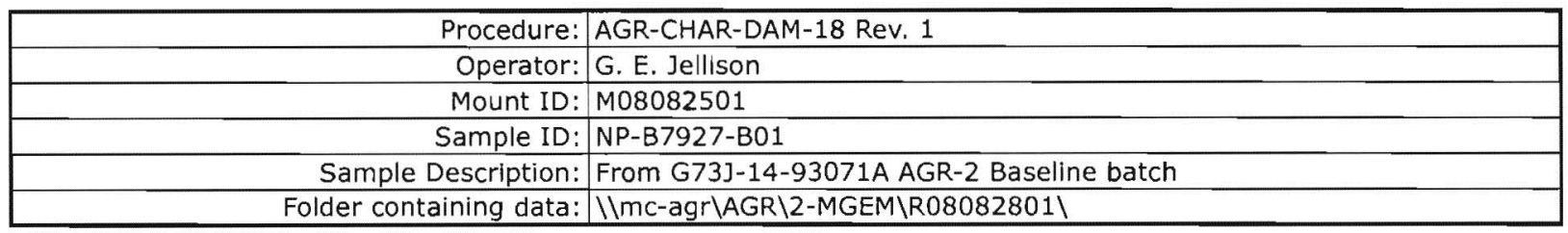

\begin{tabular}{|c|c|c|c|c|c|c|c|}
\hline \multirow{2}{*}{ Particle \# } & \multirow{2}{*}{$\begin{array}{c}\text { Grid } \\
\text { Position }\end{array}$} & \multicolumn{3}{|c|}{ Diattenuation } & \multicolumn{3}{|c|}{ True BAFo $=(1+\mathrm{N}) /(1-\mathrm{N})$} \\
\hline & & Average & St. Dev. & Ave. Error & Average & St, Dev. & Ave, Error \\
\hline 1 & 4,4 & 0.0127 & 0.0023 & 0.0004 & 1.0257 & 0.0047 & 0.0008 \\
\hline 2 & 4,5 & 0.0116 & 0.0022 & 0.0004 & 1.0235 & 0.0045 & 0.0008 \\
\hline 3 & 4,6 & 0.0130 & 0.0021 & 0.0004 & 1.0263 & 0.0043 & 0.0008 \\
\hline 4 & 5,3 & 0.0141 & 0.0022 & 0.0004 & 1.0286 & 0.0045 & 0.0008 \\
\hline 5 & 5,5 & 0.0133 & 0.0025 & 0.0005 & 1.0270 & 0.0051 & 0.0010 \\
\hline 6 & 5,6 & 0.0123 & 0.0024 & 0.0005 & 1.0249 & 0.0049 & 0.0010 \\
\hline 7 & 6,4 & 0.0131 & 0.0024 & 0.0005 & 1.0265 & 0.0049 & 0.0010 \\
\hline 8 & 6,5 & 0.0131 & 0.0023 & 0.0005 & 1.0265 & 0.0047 & 0.0010 \\
\hline 9 & 6,6 & 0.0132 & 0.0025 & 0.0004 & 1.0268 & 0.0051 & 0.0008 \\
\hline 10 & 5,7 & 0.0121 & 0.0024 & 0.0005 & 1.0245 & 0.0049 & 0.0010 \\
\hline \multicolumn{2}{|c|}{ Average } & 0.0129 & 0.0023 & 0.0005 & 1.0260 & 0.0048 & 0.0009 \\
\hline
\end{tabular}

Mean of average BAFo per particle: 1.0260 Standard deviation of average BAFo per particle: 0.0014

\section{Comments}




\section{Data Report Form DRF-18B: Measurement of Pyrocarbon Anisotropy using the 2-MGEM - OPyC}

\begin{tabular}{|c|c|}
\hline Procedure: & AGR-CHAR-DAM-18 Rev. 1 \\
\hline Operator: & G. E. Jellison \\
\hline Mount ID: & M08082501 \\
\hline Sample ID: & NP-B7927-B01 \\
\hline Sample Description: & From G73]-14-93071A AGR-2 Baseline batch \\
\hline Folder containing data: & 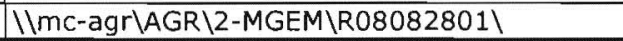 \\
\hline
\end{tabular}

\begin{tabular}{|c|c|c|c|c|c|c|c|}
\hline \multirow{2}{*}{ Particle \# } & \multirow{2}{*}{$\begin{array}{c}\text { Grid } \\
\text { Position }\end{array}$} & \multicolumn{3}{|c|}{ Diattenuation } & \multicolumn{3}{|c|}{ True BAFo $=(1+\mathrm{N}) /(1-\mathrm{N})$} \\
\hline & & Average & St. Dev. & Ave. Error & Average & St. Dev. & Ave. Error \\
\hline 1 & 4,4 & 0.0089 & 0.0021 & 0.0004 & 1.0180 & 0.0043 & 0.0008 \\
\hline 2 & 4,5 & 0.0088 & 0.0020 & 0.0004 & 1.0178 & 0.0041 & 0.0008 \\
\hline 3 & 4,6 & 0.0079 & 0.0020 & 0.0004 & 1.0159 & 0.0041 & 0.0008 \\
\hline 4 & 5,3 & 0.0088 & 0.0020 & 0.0004 & 1.0178 & 0.0041 & 0.0008 \\
\hline 5 & 5,5 & 0.0086 & 0.0023 & 0.0005 & 1.0173 & 0.0047 & 0.0010 \\
\hline 6 & 5,6 & 0.0087 & 0.0022 & 0.0005 & 1.0176 & 0.0045 & 0.0010 \\
\hline 7 & 6,4 & 0.0087 & 0.0027 & 0.0005 & 1.0176 & 0.0055 & 0.0010 \\
\hline 8 & 6,5 & 0.0097 & 0.0021 & 0.0004 & 1.0196 & 0.0043 & 0.0008 \\
\hline 9 & 6,6 & 0.0086 & 0.0021 & 0.0005 & 1.0173 & 0.0043 & 0.0010 \\
\hline 10 & 5,7 & 0.0085 & 0.0020 & 0.0005 & 1.0171 & 0.0041 & 0.0010 \\
\hline \multicolumn{2}{|c|}{ Average } & 0.0087 & 0.0022 & 0.0005 & 1.0176 & 0.0044 & 0.0009 \\
\hline
\end{tabular}

Mean of average BAFo per particle: 1.0176

Standard deviation of average BAFo per particle: 0.0009

\section{Comments}
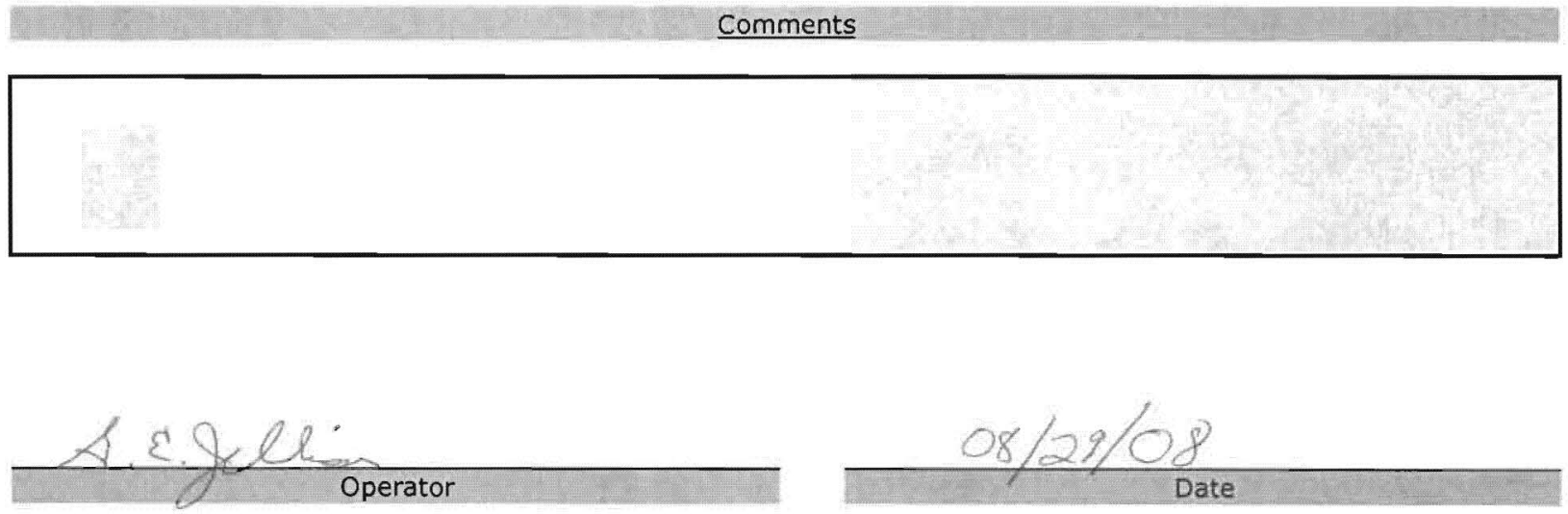
Data Report Form DRF-22: Estimation of Average Particle Weight

Procedure: AGR-CHAR-DAM-22 Rev. 1

Operator: Dixie Barker

Particle Lot ID: NP-B7927-C01

Particle Lot Description: From G73]-14-93071A AGR-2 Baseline Batch

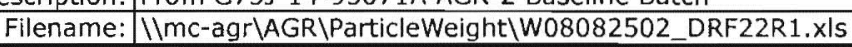

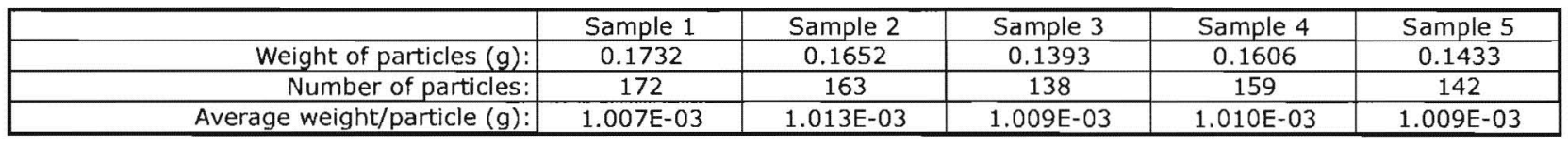

Mean average weight/particle (g): $1.010 \mathrm{E}-03$

$\begin{array}{ll}\text { Standard error in mean average weight/particle }(\mathrm{g}): & 1.06 \mathrm{E}-06\end{array}$
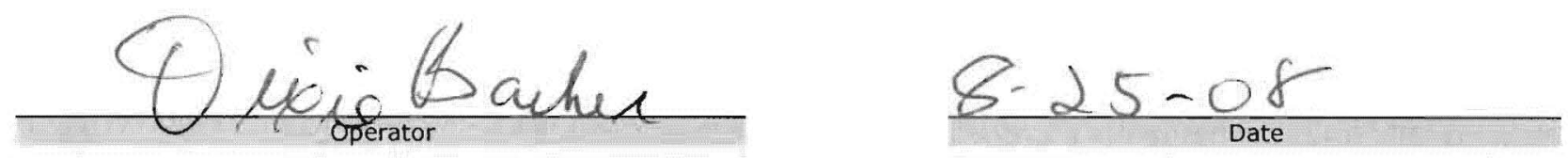


\section{Data Report Form DRF-31: Measurement of Open Porosity using a Mercury Porosimeter}

\begin{tabular}{|c|c|}
\hline Procedure: & AGR-CHAR-DAM-31 Rev. 1 \\
\hline Operator: & S. D. Nunn \\
\hline Coated particle batch ID: & NP-B7927-D01 \\
\hline Batch Description: & From G73J-14-93071A AGR-2 Basellne batch \\
\hline Thermocouple Expiration Date: & $5 / 15 / 09$ \\
\hline Penetrometer Expiration Date: & $7 / 10 / 09$ \\
\hline Completed DRF Filename: & 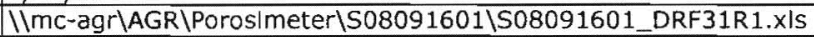 \\
\hline
\end{tabular}

Mean average weight/particle $(\mathrm{g}): 1.01 \mathrm{E}-03$

$\begin{array}{ll}\text { Standard error in mean average welght/particle }(\mathrm{g}): & 1.06 \mathrm{E}-06\end{array}$

\begin{tabular}{|r|l|}
\hline Weight of particles $(\mathrm{g}):$ & 3.8896 \\
\hline Approximate number of particles: & 3851 \\
\hline Uncertalnty in number of particles: & 4 \\
\hline Total envelope volume of sample $(\mathrm{cc}):$ & 1.284 \\
\hline Average envelope volume/particle $(\mathrm{cc}):$ & $3.33 \mathrm{E}-04$ \\
\hline Sample envelope density $(\mathrm{g} / \mathrm{cc}):$ & 3.029 \\
\hline
\end{tabular}

Average particle diameter (microns): $8.60 \mathrm{E}+02$

Average surface area/particle $(\mathrm{cm} 2): 2.33 \mathrm{E}-02$

Total sample surface area $(\mathrm{cm} 2): 8.96 \mathrm{E}+01$

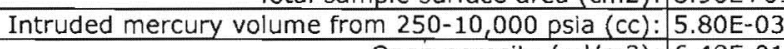

Open porosity $(\mathrm{ml} / \mathrm{m} 2): 6.48 \mathrm{E}-01$

S.D. $)(4,2, n$

Operator
$9 / 16 / 08$

Date 


\section{Data Report Form DRF-32: Counting of Particles with SiC Soot Inclusion Defects by Visual Inspection}

\begin{tabular}{|c|c|}
\hline Procedure: & AGR-CHAR-DAM-32 Rev. 0 \\
\hline Operator: & Fred Montgomery \\
\hline Sample ID: & NP-B7927-E01 \\
\hline Sample Description: & from G73]-14-93071A AGR-2 Baseline batch \\
\hline Folder containing images: & 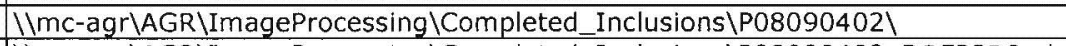 \\
\hline
\end{tabular}

\begin{tabular}{|r|l|}
\hline Mean average weight/particle $(\mathrm{g}):$ & $1.01 \mathrm{E}-03$ \\
\hline Uncertainty in average weight/particle $(\mathrm{g}):$ & $1.06 \mathrm{E}-06$ \\
\hline Weight of sample of particles $(\mathrm{g}):$ & 4.941 \\
\hline Approximate number of particles in sample: & 4892 \\
\hline Uncertainty in number of particles in sample: & 5 \\
\hline
\end{tabular}

Number of particles with SiC soot inclusion defects: 25

\section{Comments}

$25 / 4892$ corresponds to $<7.2 \mathrm{E}-3$ defect fraction at $95 \%$ confidence.

Many of the defects were marginal and possibly related to porosity bands. Dark field rings were much more prevalent in this sample than in the qualification batches, indicating a change in the SiC coating conditions.

There are 9 particles in this population that have sufficiently large inclusions in the SiC that it changes the radius of curvature and forms a bulge in the SiC. These are of greater concern and represent a defect population of $<3.3 \mathrm{E}-3$ at $95 \%$ confidence.

The most serious defect is a particle missing a portion of the IPyC layer. This could represent a defect population as high as 9.7E-4. 
NP-B7927-E01, G73J-14-93071A AGR-2 Baseline batch.

The observed defect fraction was $25 / 4892$, this corresponds to $<7.2 \mathrm{E}-3$ defect fraction at $95 \%$ confidence. Many of the defects were marginal and possibly related to porosity bands. Dark field rings were much more prevalent in this sample than in the qualification batches, indicating a change in the $\mathrm{SiC}$ coating conditions.

There were 9 particles in this population that had sufficiently large inclusions in the $\mathrm{SiC}$ that it changed the radius of curvature and formed a bulge in the SiC. These are of greater concern and represent a defect population of $<3.3 \mathrm{E}-3$ at $95 \%$ confidence. Images are provided below.

A major issue with the soot inclusions observed is that over half only cover small arcs. This contradicts the assumption that a single polish plane is sufficient to do this analysis and means that the defect fraction may be higher than 3.3E-3, which was calculated by a simple binomial distribution. The single polish plane assumption was based on observations of earlier batches that had larger inclusions, but this particular batch is showing a different fluidization problem, probably related to the presence of the hot sample cup in the bed.

The most serious defect is a particle missing a portion of the IPyC layer, possibly broken off when the cup dropped or due to subsequent collision with the cup. This could represent a defect population as high as 9.7E-4. Notice that it also appears in the image below that the kernel surface may have suffered attack by chlorine.

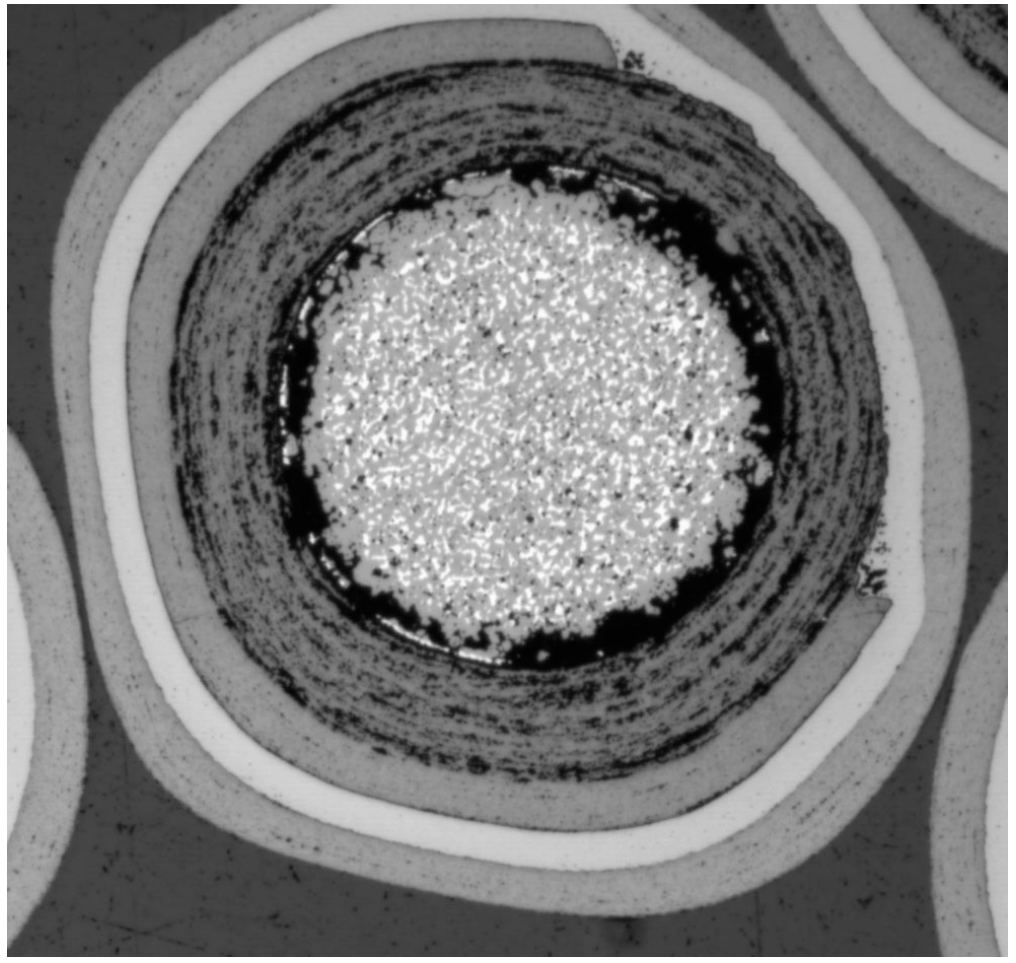

P0809040204 XYF022 


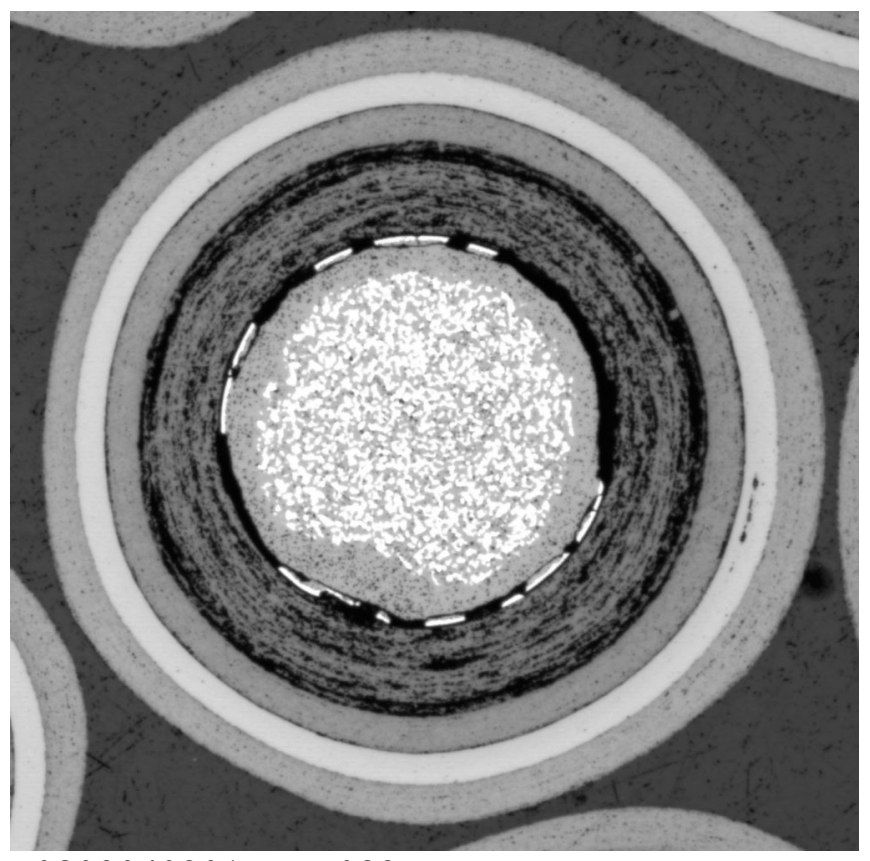

P0809040201 XYF083

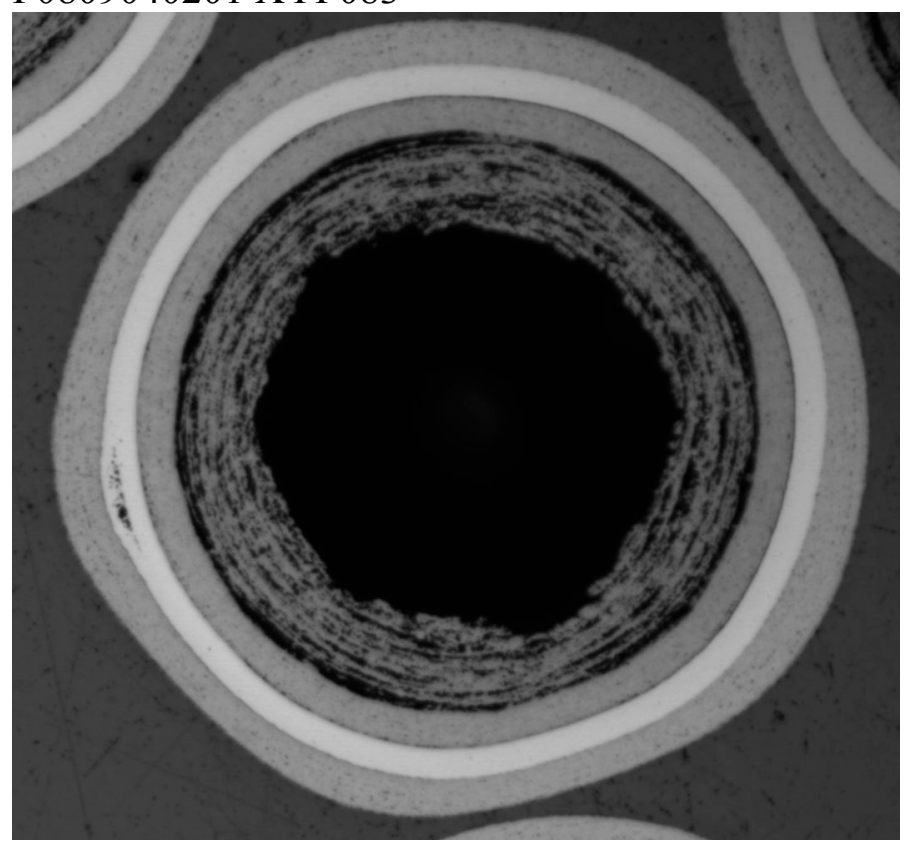

P0809040202 XYF087

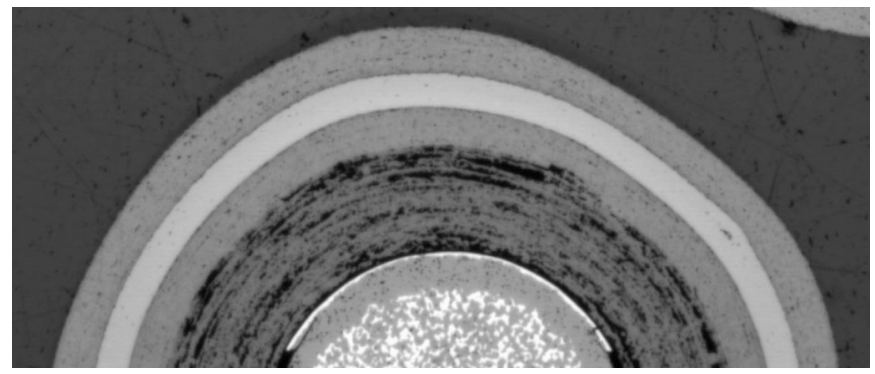

P0809040203 XYF023 


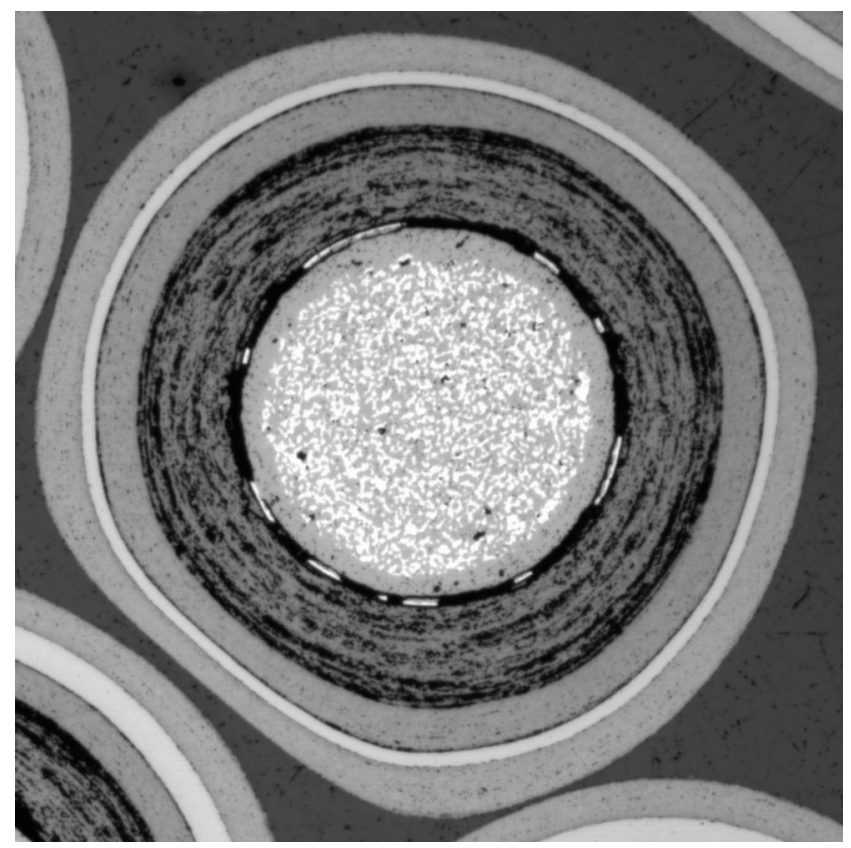

P0809040204 XYF029

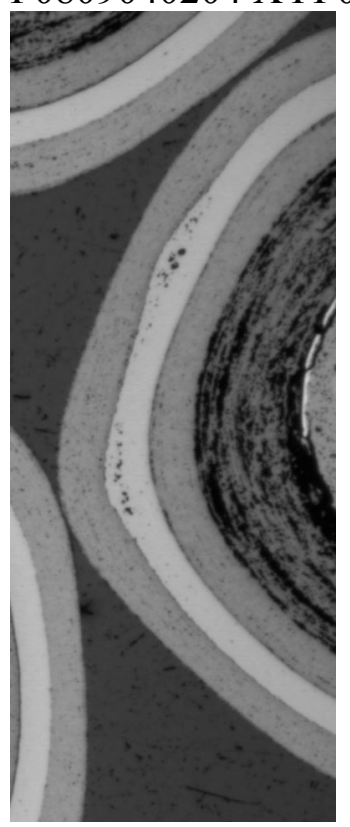

P0809040204 XYF032

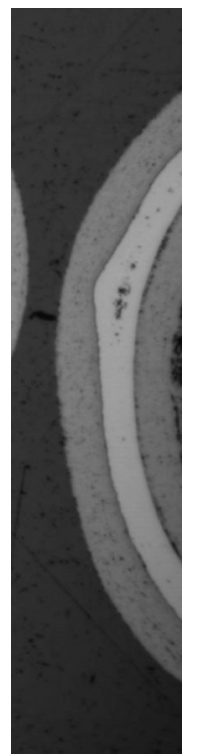

P0809040204 XYF036

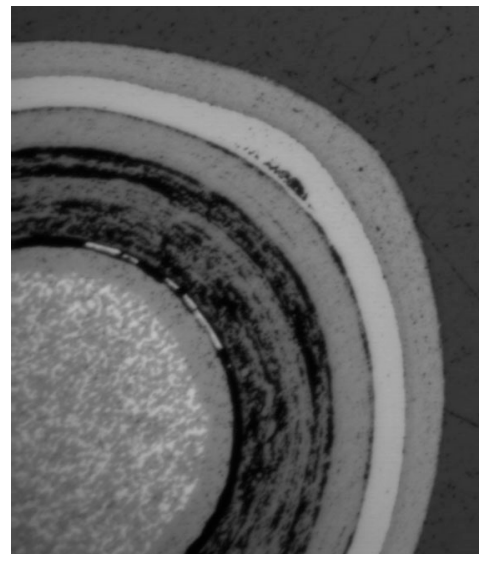

P0809040204 XYF058 


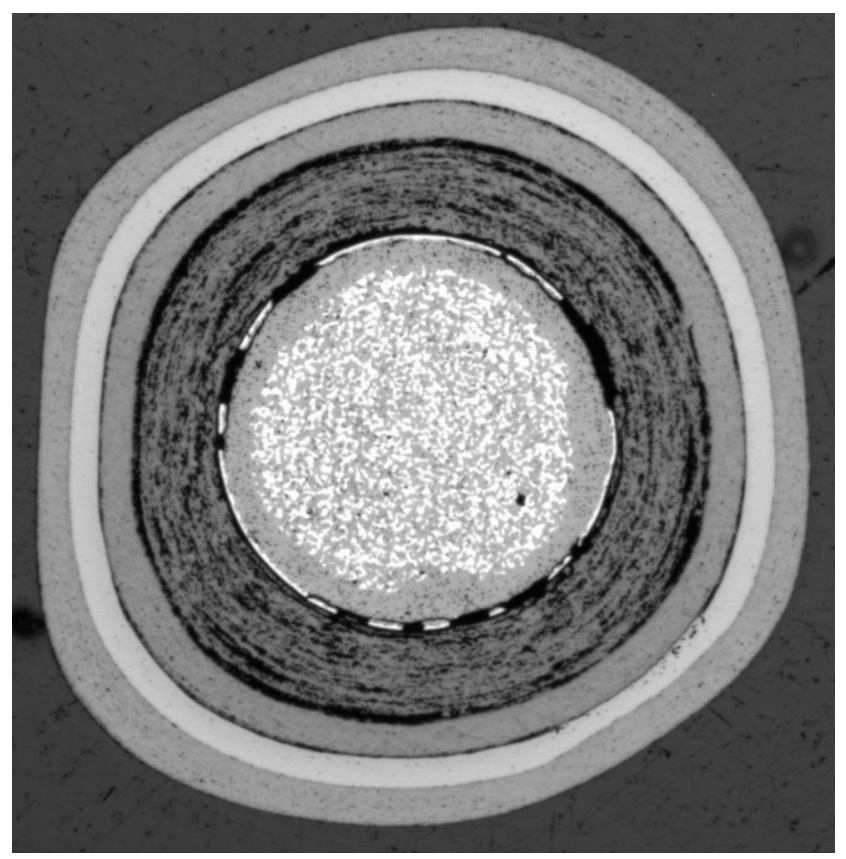

P0809040205 XYF011

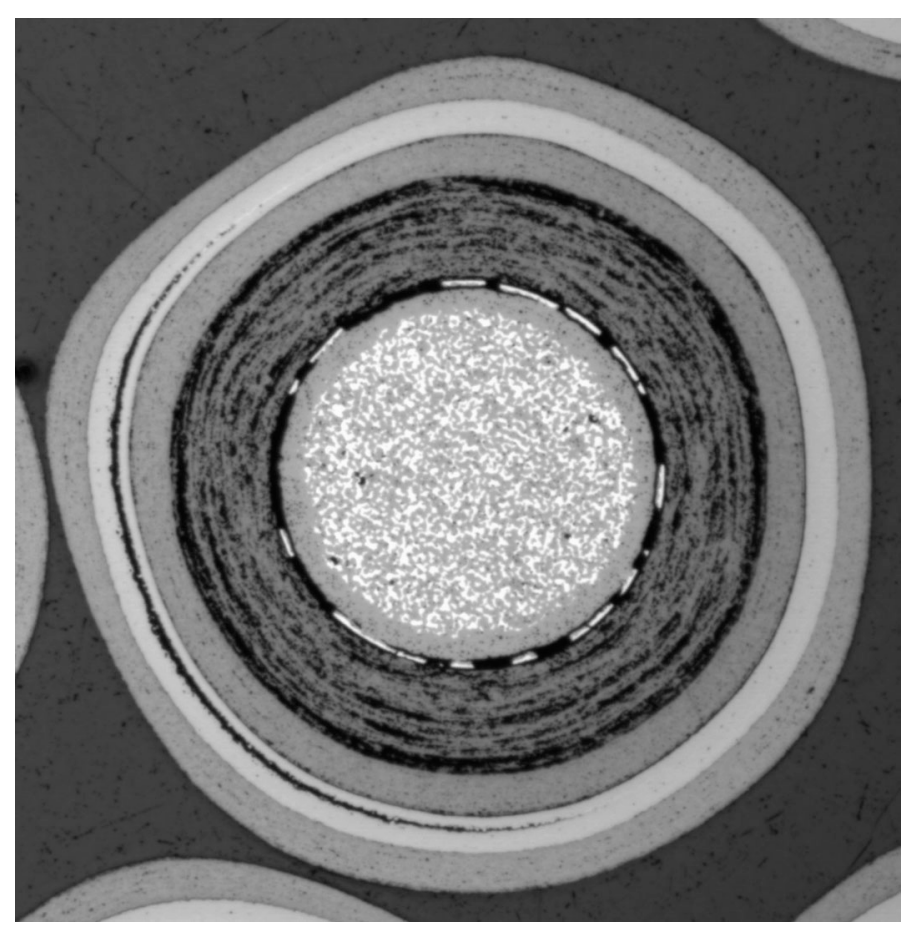

P0809040205 XYF055 